Research Article

\title{
Existence Theory and Novel Iterative Method for Dynamical System of Infectious Diseases
}

\author{
Gauhar Ali, ${ }^{1}$ Ghazala Nazir, ${ }^{1}$ Kamal Shah $\mathbb{D}^{1},{ }^{1}$ and Yongjin Li $\mathbb{C D}^{2}$ \\ ${ }^{1}$ Department of Mathematics, University of Malakand, Chakdara, Dir (Lower), Khyber Pakhtunkhwa, Pakistan \\ ${ }^{2}$ Department of Mathematics, Sun Yat-sen University, Gangzhou, China \\ Correspondence should be addressed to Yongjin Li; stslyj@mail.sysu.edu.cn
}

Received 24 February 2020; Revised 30 April 2020; Accepted 13 May 2020; Published 1 June 2020

Academic Editor: Emilio Jiménez Macías

Copyright (c) 2020 Gauhar Ali et al. This is an open access article distributed under the Creative Commons Attribution License, which permits unrestricted use, distribution, and reproduction in any medium, provided the original work is properly cited.

This manuscript is devoted to investigate qualitative theory of existence and uniqueness of the solution to a dynamical system of an infectious disease known as measles. For the respective theory, we utilize fixed point theory to construct sufficient conditions for existence and uniqueness of the solution. Some results corresponding to Hyers-Ulam stability are also investigated. Furthermore, some semianalytical results are computed for the considered system by using integral transform due to the Laplace and decomposition technique of Adomian. The obtained results are presented by graphs also.

\section{Introduction}

In recent time, the subject of fractional calculus has got much attention from the researcher. This is due to large numbers of applications in various disciplines of science and engineering where the concept of derivatives and integrals is frequently used. Normally, the integer-order derivative does not explore the dynamics of real-world problems more comprehensively as compared to the fractional derivative. Also, the fractional differential operator is global and poses greater degree of freedom, while the ordinary differential operator is local and often cannot explain the memory and hereditary process of the real-world problem more efficiently. In fact, fractional derivative is definite integral over a domain; therefore, it has been defined in many ways. In this regard, a variety of definitions have been introduced by different researchers in the literature. Some famous definitions are those given by Riemann and Liouville, Caputo, Hadamard, and so on (see [1]). The definitions of Riemann-Liouville and Caputo have been very well used in applied problems. These definitions involve singular kernel which often causes difficulty in dealing some problems. Therefore, Caputo and Fabrizio in 2015 introduced a new concept about fractional-order derivatives based on nonsingular kernel (see [2-4]). Some remarkable merits of
Caputo-Fabrizio fractional derivative and integral and their applications were given by many researchers in previous few years (see, for detail, [5-13]). The concerned fractional integral of a function is the fractional average of the function itself and its fractional integral in Riemann-Liouville sense. Moreover, in some articles, it has been displayed that the derivative has some constructive applications in thermal science, material sciences, and so on (see, for detail, $[11,12,14,15])$. Since differential operators have greater degree of freedom, therefore, to find the exact solution to each and every problem is quite difficult. In this regard, great motivation has been observed in the last two decades to establish best tools to handle such problems. One of the important techniques is to find analytical approximate solutions to many nonlinear problems of FODEs. For this purpose, the usual decompositions, perturbation, and integral transform methods were greatly utilized to investigate ordinary differential and integral equations. The mentioned techniques have been very well explored for fractional differential equations (see [16-25]). One of the powerful methods which has been used very frequently in the past is due to the Laplace Adomian decomposition method. For usual FODEs, the mentioned method has been used very regularly in the literature. However, to the best of our information, the aforesaid method is very rarely used for FODEs involving nonsingular kernel, see [26, 27]. 
Here, we remark that mathematical models are the powerful tools to study various dynamical problems of physical and biological sciences. The concept was initiated by Bernoulli in 1776. However, a formal mathematical model of three compartments was constructed in 1927 by McKendrick and his co-author called the SIR mode. Later on, the subject of mathematical modeling was extended to infectious diseases. Because mathematical models of the biological problem have become powerful tools to understand various infectious diseases, the proper method has to be developed to control the disease or minimize its transmission in the society. From ancient time, measles disease is one of the most threatful diseases. It was a big reason for children death in the past. This dangerous disease was caused by germs called morbilliform. Measles disease spreeds, when an infected person coughs or sneezes, because its virus can live for up to two hours in an airspace where the infected person coughed or sneezed. Measles-infected individuals can transfer their germs to the other people 4-8 days before and after the skin eruptions start. It transmits a disease in young babies up to 30-40 million every year. Measles appears once and is present for a long time in life for immunity. Symptoms of the disease include runny nose, high temperature, coughing, and spots on the whole body and in highly complicated cases, ear infections, diarrhea, and pneumonia. The vaccination of measles has been used to control disease in kids. Some vaccinated individuals could remain susceptible individuals when vaccination gets failed. Worldwide vaccination reduced $80 \%$ death caused by measles between 2000 and 2017. However, measles disease is still familiar disease in highly developing countries of Asia and Africa particularly due to the lack of proper treatment of this infectious disease. For this purpose, a massive research has been carried out to enhance the understanding of the virus of measles dynamics in various areas. For example, the authors in [28-33] discussed the global stability of the model with five compartments as

$$
\left\{\begin{array}{l}
\frac{\mathrm{d} u}{\mathrm{~d} t}=\Lambda(1-q)-\beta u(x+y)+\gamma v-\mu u \\
\frac{\mathrm{d} v}{\mathrm{~d} t}=\Lambda(q)+\mu u-\gamma x-\mu x-\omega x \\
\frac{\mathrm{d} x}{\mathrm{~d} t}=\beta u(v+y)-\mu v-\alpha v-\mu_{2} v \\
\frac{\mathrm{d} y}{\mathrm{~d} t}=\alpha v+\delta y-\mu y-\mu_{3} y \\
\frac{\mathrm{d} z}{\mathrm{~d} t}=\mu_{2} v+\omega x+\mu_{3} x-\mu z
\end{array}\right.
$$

where $(u)$ represents the susceptible, $(v)$ represents the vaccinated, $(x)$ represents the exposed, $(y)$ represents the infected, and $(z)$ represents the recovered individuals. The description of the parameters is given in the analytical section. These models have been investigated corresponding to ordinary and usual fractional-order derivatives. Furthermore, the researchers have given the global and local dynamics by computing the basic reproductive numbers. Here, we investigate the given model under the nonsingular fractional derivative of Caputo and Fabrizio (CFFD) from other perspectives including the qualitative analysis by using fixed point approach. Further stability is a required aspect in the dynamical problem. Since we are going to derive approximate solutions, therefore, in this regard, Hyers-Ulamtype stability results are investigated. The mentioned stability has been very well studied for the general problem of FODEs, see, for detail, [5, 34-36]. Also, the analytical results are investigated through the Laplace Adomian decomposition method. We considered model (1) under the CFFD with fractional order $\eta \in(0,1]$ as

$$
\left\{\begin{array}{l}
{ }^{C F} D_{t}^{\eta}(u)(t)=\Lambda(1-q)-\beta u(v+y)+\gamma v-\mu u \\
{ }^{C F} F D_{t}^{\eta}(v)(t)=\Lambda(q)+\mu u-\gamma x-\mu x-\omega x \\
{ }^{C F} F D_{t}^{\eta}(x)(t)=\beta u(v+y)-\mu v-\alpha v-\mu_{2} v \\
{ }^{C F} F D_{t}^{\eta}(y)(t)=\alpha v+\delta y-\mu y-\mu_{3} y \\
{ }^{C F} F D_{t}^{\eta}(z)(t)=\mu_{2} v+\omega x+\mu_{3} x-\mu z
\end{array}\right.
$$

under the initial conditions

$$
\begin{aligned}
& u(0) \geq 0, \\
& v(0) \geq 0, \\
& x(0) \geq 0, \\
& y(0) \geq 0, \\
& z(0) \geq 0
\end{aligned}
$$

We obtain the solution in the form of series for the considered problem. Also, we display the results against different values of fractional order $\eta \in(0,1]$. Also, we provide results about the existence and uniqueness of the solution for the concerned model by using fixed point theorems due to Schauder and Banach. Here, we remark that we use the Laplace Adomian decomposition method because this method is easy and efficient and less expensive. Furthermore, the mentioned method does not require any predefine step size or controlling parameter which are needed by RK methods or the homotopy method, respectively, for detail, see [37-39]. Furthermore, the convergence of the method has been showed in many papers of the proposed method, for instance, see [27]. 


\section{Preliminaries}

Definition 1 (see [3]). Let $\varphi \in \mathscr{H}^{1}\left(a_{1}, a_{2}\right), a_{2}>a_{1}$, and $\eta \in(0,1)$; then, the CFFD is recalled as

$$
{ }^{C F} \mathscr{D}_{t}^{\eta}(\varphi(t))=\frac{\mathscr{M}(\eta)}{1-\eta} \int_{a}^{t} \varphi^{\prime}(t) \exp \left[-\eta \frac{t-\xi}{1-\eta}\right] \mathrm{d} \xi .
$$

$\mathscr{M}(\eta)$ is the normalization function defined as $\mathscr{M}(\eta)=$ $(2 \eta /(2-\eta))$ and satisfies the conditions $\mathscr{M}(1)=\mathscr{M}(0)=1$. If the function fails to exist in $\mathscr{H}^{1}\left(a_{1}, a_{2}\right)$, then the derivative can be redefined as

$$
{ }^{C F} \mathscr{D}_{t}^{\eta}(\varphi(t))=\frac{\mathscr{M}(\eta)}{1-\eta} \int_{a}^{t}(\varphi(t)-\varphi(\xi)) \exp \left[-\eta \frac{t-\xi}{1-\eta}\right] \mathrm{d} \xi .
$$

Definition 2 (see [3]). The integral of fractional order $\eta \in(0,1]$ of a function $\varphi$ is defined by

$$
{ }_{0}^{C F} \mathscr{F}_{t}^{\eta}[\varphi(t)]=\frac{(1-\eta)}{\mathscr{M}(\eta)} \varphi(t)+\frac{\eta}{\mathscr{M}(\eta)} \int_{0}^{t} \varphi(\xi) \mathrm{d} \xi, \quad t \geq 0 .
$$

Lemma 1 (see [40]). The solution of the differential equation involving CFFD for $y \in L[0, T]$ as

$$
\begin{aligned}
& { }^{C F} \mathscr{D}_{t}^{\eta}(\varphi(t))=y(t), \quad 0<\eta \leq 1, \\
& \varphi(0)=\varphi_{0}, \varphi \text { is any real constant, }
\end{aligned}
$$

is given by

$$
\varphi(t)=\varphi_{0}+\frac{(1-\eta)}{\mathscr{M}(\eta)}[y(t)-y(0)]+\frac{\eta}{\mathscr{M}(\eta)} \int_{0}^{t} y(\xi) \mathrm{d} \xi .
$$

Definition 3 (see $[34,35]$ ). The solution $\varphi \in C[0, T]$ of the differential equation

$$
\begin{aligned}
& { }^{C F} \mathscr{D}_{t}^{\eta}(\varphi(t))=y(t), \quad 0<\eta \leq 1, \\
& \varphi(0)=\varphi_{0}, \varphi \text { is any real constant, }
\end{aligned}
$$

is Hyers-Ulam stable; if there exists $\varepsilon>0$ such that, for the inequality

$$
\left|{ }^{C F} \mathscr{D}_{t}^{\eta}(\varphi(t))-y(t)\right| \leq \varepsilon, \quad t \in[0, T],
$$

there exists a unique solution $\bar{\varphi} \in C[0, T]$ with a constant $C_{y}>0$ such that

$$
|\varphi(t)-\bar{\varphi}(t)| \leq C_{y} \varepsilon, \quad t \in[0, T] .
$$

Furthermore, if there exists nondecreasing function $g:(0,1) \longrightarrow \mathscr{R}^{+}$such that (11) may be written as

$$
|\varphi(t)-\bar{\varphi}(t)| \leq C_{y} g(\varepsilon), \quad \text { with } g(0)=0,
$$

then the concerned solution of problem (9) is generalized Hyers-Ulam stable.
Theorem 1 (see [40]). Let B be a convex subset of Banach space $\mathbf{X}$, with operators $\mathbf{G}$ and $\mathbf{H}$ with

(1) $\mathbf{G}(u)+\mathbf{H}(v) \in \mathbf{B}$ for all $u, v \in \mathbf{B}$

(2) $\mathbf{G}$ is the condensing operator

(3) $\mathbf{H}$ is continues and compact

Then, there exists at least one solution $u \in \mathbf{B}$ such that

$$
\mathbf{G}(u)+\mathbf{H}(u)=u .
$$

Definition 4 (see $[4,14]$ ). The Laplace transform of CFFD ${ }_{0}^{C F} \mathscr{D}_{t}^{\eta} x(t)$ is given as

$$
\mathscr{L}\left[{ }_{0}^{C F} \mathscr{D}_{t}^{\eta} x(t)\right]=\frac{s \mathscr{L}[x(t)]-x(0)}{s+\eta(1-s)}, \quad s \geq 0, \eta \in(0,1] .
$$

\section{Existence and Stability Results for the Considered Model}

In this part of the manuscript, we determine existence results for model (2) using the fixed point theorem due to Banach. In this regard, we first define the following functions:

$$
\begin{aligned}
& \varphi_{1}(t, u, v, x, y, z)=\Lambda(1-q)-\beta u(x+y)+\gamma v-\mu u, \\
& \varphi_{2}(t, u, v, x, y, z)=\Lambda(q)+\mu u-\gamma x-\mu x-\omega x, \\
& \varphi_{3}(t, u, v, x, y, z)=\beta u(v+y)-\mu v-\alpha v-\mu_{2} v, \\
& \varphi_{4}(t, u, v, x, y, z)=\alpha v+\delta y-\mu y-\mu_{3} y, \\
& \varphi_{5}(t, u, v, x, y, z)=\mu_{2} v+\omega x+\mu_{3} x-\mu z .
\end{aligned}
$$

Then, we write some notions for easiness as

$$
\begin{aligned}
\mathbf{Y}(t) & =\left\{\begin{array}{l}
u(t) \\
v(t) \\
x(t), \\
y(t) \\
z(t)
\end{array}\right. \\
\mathbf{Y}_{0} & =\left\{\begin{array}{l}
u(0) \\
v(0) \\
x(0), \\
y(0) \\
z(0)
\end{array}\right. \\
\Psi(t, \mathbf{Y}(t)) & =\left\{\begin{array}{l}
\varphi_{1}(t, u, v, x, y, z) \\
\varphi_{2}(t, u, v, x, y, z) \\
\varphi_{3}(t, u, v, x, y, z), \\
\varphi_{4}(t, u, v, x, y, z) \\
\varphi_{5}(t, u, v, x, y, z)
\end{array}\right.
\end{aligned}
$$




$$
\Psi_{0}=\left\{\begin{array}{l}
\varphi_{1}(0, u(0), v(0), x(0), y(0), z(0)) \\
\varphi_{2}(0, u(0), v(0), x(0), y(0), z(0)) \\
\varphi_{3}(0, u(0), v(0), x(0), y(0), z(0)) \\
\varphi_{4}(0, u(0), v(0), x(0), y(0), z(0)) \\
\varphi_{5}(0, u(0), v(0), x(0), y(0), z(0))
\end{array}\right.
$$

Using (16), system (2) can be written as

$$
{ }^{C F} D_{t}^{\eta}[\mathbf{Y}(t)]=\Psi(t, \mathbf{Y}(t)), \quad t \in[0, T], \mathbf{Y}(0)=\mathbf{Y}_{0} \text {. }
$$

In view of Lemma 1 , problem (18) can be converted to the given integral equation as

$$
\begin{aligned}
\mathbf{Y}(t)= & \mathbf{Y}_{0}+\left[\Psi(t, \mathbf{Y}(t))-\Psi_{0}\right] \frac{1-\eta}{\mathscr{M}(\eta)} \\
& +\frac{\eta}{\mathscr{M}(\eta)} \int_{0}^{t} \Psi(\xi, \mathbf{Y}(\xi)) \mathrm{d} \xi
\end{aligned}
$$

Now, let $0 \leq t \leq T<\infty$, and let $\mathscr{J}=[0, T]$; we define the Banach space $\mathbf{X}=C\left([0, T] \times \mathscr{R}^{5}, \mathscr{R}\right)$ under the norm

$$
\|\mathbf{Y}\|=\sup _{t \in \mathscr{J}}\{|\mathbf{Y}|: \mathbf{Y} \in \mathbf{X}\} .
$$

The assumptions given in the following hold true:

$\left(\mathscr{A}_{1}\right)$ Under the continuity of $\Psi: \mathscr{J} \times \mathscr{R}^{5} \longrightarrow \mathscr{R}$, there exists $K_{\Psi}>0$ such that

$$
|\Psi(t, \mathbf{Y}(t))-\Psi(t, \overline{\mathbf{Y}}(t))| \leq K_{\Psi}|\mathbf{Y}(t)-\overline{\mathbf{Y}}(t)|
$$

$\left(\mathscr{A}_{2}\right)$ There exists positive constant $C_{\Psi}, M_{\Psi}>0$ such that $|\Psi(t, \mathbf{Y}(t))| \leq C_{\Psi}|\mathbf{Y}|+M_{\Psi}$.

Theorem 2. Under the assumptions $\left(\mathscr{A}_{1}\right),\left(\mathscr{A}_{2}\right)$, problem (19) has at least one solution if $K_{\Psi} \mid<\mathscr{M}(\eta)$; consequently, the considered system (2) has at least one solution.

Proof. Let $\mathbf{B}=\{\mathbf{Y} \in X:\|\mathbf{Y}\| \leq \rho, \rho>0\} \subset X$ be a closed convex set. Now, we define the operators from (19) as

$$
\begin{aligned}
& \mathbf{G Y}(t)=\mathbf{Y}_{0}+\left[\Psi(t, \mathbf{Y}(t))-\Psi_{0}\right] \frac{(1-\eta)}{\mathscr{M}(\eta)}, \\
& \mathbf{H Y}(t)=\frac{\eta}{\mathscr{M}(\eta)} \int_{0}^{t} \Psi(\xi, \mathbf{Y}(\xi)) \mathrm{d} \xi .
\end{aligned}
$$

To derive the required results, we first prove that operator $\mathbf{G}: \mathbf{B} \longrightarrow \mathbf{B}$ is a contraction.

For any $\mathbf{Y}, \overline{\mathbf{Y}} \in \mathbf{B}$, one has

$$
\begin{aligned}
\|\mathbf{G Y}-\mathbf{G} \overline{\mathbf{Y}}\|= & \sup _{t \in \mathcal{F}} \mid\left[\mathbf{Y}_{0}+\left[\Psi(t, \mathbf{Y}(t))-\Psi_{0}\right] \frac{(1-\eta)}{\mathscr{M}(\eta)}\right] \\
& -\left[\mathbf{Y}_{0}+\left[\Psi(t, \overline{\mathbf{Y}}(t))-\Psi_{0}\right] \frac{(1-\eta)}{\mathscr{M}(\eta)}\right] \mid \\
\leq & \frac{K_{\Psi}}{\mathscr{M}(\eta)}\|\mathbf{Y}-\overline{\mathbf{Y}}\| .
\end{aligned}
$$

This shows that $\mathbf{G}$ is a contraction. Now, to show that $\mathbf{H}: \mathbf{B} \longrightarrow \mathbf{B}$ is bounded and equicontinuous, the continuity of $\Psi$ implies that $\mathbf{H}$ is continuous. For any $\mathbf{Y} \in \mathbf{B}$, we have

$$
\begin{aligned}
\|\mathbf{H Y}\| & =\sup _{t \in \mathcal{J}}\left|\frac{\eta}{\mathscr{M}(\eta)} \int_{0}^{t} \Psi(\xi, \mathbf{Y}(\xi)) \mathrm{d} \xi\right| \\
& \leq \frac{\eta}{\mathscr{M}(\eta)}\left(C_{\Psi}|\mathbf{Y}|+M_{\psi}\right) \int_{0}^{T} \mathrm{~d} \xi \\
& \leq \frac{T}{\mathscr{M}(\eta)}\left(C_{\Psi} \rho+M_{\psi}\right) .
\end{aligned}
$$

This shows that $\mathbf{H}$ is bounded; for equicontinuity, let $t_{1}>t_{2}$, and we have

$$
\begin{aligned}
\left|\mathbf{H Y}\left(t_{1}\right)-\mathbf{H Y}\left(t_{2}\right)\right|= & \frac{\eta}{\mathscr{M}(\eta)} \mid \int_{0}^{t_{1}} \Psi(\xi, \mathbf{Y}(\xi)) \mathrm{d} \xi \\
& -\int_{0}^{t_{2}} \Psi(\xi, \mathbf{Y}(\xi)) \mathrm{d} \xi \mid \\
\leq & \frac{\left(C_{\Psi} \rho+M_{\psi}\right)}{\mathscr{M}(\eta)}\left[t_{1}-t_{2}\right],
\end{aligned}
$$

which implies that $\left|\mathbf{H Y}\left(t_{1}\right)-\mathbf{H Y}\left(t_{2}\right)\right| \longrightarrow 0$ as $t_{1} \longrightarrow t_{2}$. So, $\mathbf{H}$ is uniformly continuous and bounded. Thus, by Arzelá-Ascoli theorem, $\mathbf{H}$ is relatively compact and so is completely continuous. Thus, by Theorem 1, problem (18) has at least one solution. Consequently, the considered model (2) has at least one solution.

Next, we define the operator T: $X \longrightarrow X$ by

$$
\begin{aligned}
\mathbf{T}(\mathbf{Y})= & \mathbf{Y}_{0}+\left[\Psi(t, \mathbf{Y}(t))-\Psi_{0}\right] \frac{1-\eta}{\mathscr{M}(\eta)} \\
& +\frac{\eta}{\mathscr{M}(\eta)} \int_{0}^{t} \Psi(\xi, \mathbf{Y}(\xi)) \mathrm{d} \xi
\end{aligned}
$$

Theorem 3. Under assumption $\left(\mathscr{A}_{2}\right)$, the operator $\mathbf{T}: X \longrightarrow X$ as defined in (26) is a contraction; then, problem (18) has a unique solution with the condition $L_{\Psi}=\left(K_{\Psi}(1+T) / \mathscr{M}(\eta)\right)<1$, and consequently, our proposed system (2) has a unique solution.

Proof. Let $\mathbf{Y}, \overline{\mathbf{Y}} \in X$; then, from (26), one has

$$
\begin{aligned}
\|\mathbf{T Y}-\mathbf{T} \overline{\mathbf{Y}}\|= & \sup _{t \in \mathscr{J}} \mid\left(\mathbf{Y}_{0}+\left[\Psi(t, \mathbf{Y}(t))-\Psi_{0}\right] \frac{(1-\eta)}{\mathscr{M}(\eta)}\right. \\
& \left.+\frac{\eta}{\mathscr{M}(\eta)} \int_{0}^{t} \Psi(\xi, \mathbf{Y}(\xi)) \mathrm{d} \xi\right) \\
& -\left(\mathbf{Y}_{0}+\left[\Psi(t, \overline{\mathbf{Y}}(t))-\Psi_{0}\right] \frac{(1-\eta)}{\mathscr{M}(\eta)}\right. \\
& \left.+\frac{\eta}{\mathscr{M}(\eta)} \int_{0}^{t} \Psi(\xi, \overline{\mathbf{Y}}(\xi)) \mathrm{d} \xi\right) \mid \\
\leq & L_{\Psi}\|\mathbf{Y}-\overline{\mathbf{Y}}\| .
\end{aligned}
$$


This shows that $\mathbf{T}$ is a contraction. Therefore, problem (18) has a unique solution. Hence, our considered system (2) has a unique solution.

Remark 1. Next, for stability analysis, we consider a small perturbation $\theta$, such that $\theta(0)=0$, depends only on the solution.

(i) $|\theta(t)|<\varepsilon$ for $\varepsilon>0$

(ii) ${ }_{0}^{C F} \mathbf{D}_{t}^{\eta} \mathbf{Y}(t)=\Psi(t, \mathbf{Y}(t))+\theta(t)$

Lemma 2. The solution of the perturbed problem

$$
\begin{aligned}
{ }_{0}^{C F} \mathbf{D}_{t}^{\eta} \mathbf{Y}(t) & =\Psi(t, \mathbf{Y}(t))+\theta(t), \\
\mathbf{Y}(0) & =\mathbf{Y}_{0},
\end{aligned}
$$

satisfies the given relation:

$$
\left|\mathbf{Y}(t)-\left(\mathbf{Y}_{0}+\left[\Psi(t, \mathbf{Y}(t))-\Psi_{0}\right] \frac{(1-\eta)}{\mathscr{M}(\eta)}+\frac{\eta}{\mathscr{M}(\eta)} \int_{0}^{t} \Psi(\xi, \mathbf{Y}(\xi)) \mathrm{d} \xi\right)\right| \leq \Delta \varepsilon,
$$

where $((1+T) / \mathscr{M}(\eta))=\Delta$.

Proof. In view of Lemma 1, the solution of perturb problem (28) is given by

$$
\mathbf{Y}(t)=\mathbf{Y}_{0}+\frac{(1-\eta)}{\mathscr{M}(\eta)}\left[\Psi(t, \mathbf{Y}(t))-\Psi_{0}\right]+\frac{\eta}{\mathscr{M}(\eta)} \int_{0}^{t} \Psi(\xi, \mathbf{Y}(\xi)) \mathrm{d} \xi+\frac{(1-\eta)}{\mathscr{M}(\eta)} \theta(t)+\frac{\eta}{\mathscr{M}(\eta)} \int_{0}^{t} \theta(\xi) \mathrm{d} \xi
$$

From (30), on using Remark 1, we have

$$
\begin{aligned}
\mid \mathbf{Y} & (t)-\left[\mathbf{Y}_{0}+\frac{(1-\eta)}{\mathscr{M}(\eta)}\left[\Psi(t, \mathbf{Y}(t))-\Psi_{0}\right]+\frac{\eta}{\mathscr{M}(\eta)} \int_{0}^{t} \Psi(\xi, \mathbf{Y}(\xi)) \mathrm{d} \xi \mid\right. \\
& =\left|\frac{(1-\eta)}{\mathscr{M}(\eta)} \theta(t)+\frac{\eta}{\mathscr{M}(\eta)} \int_{0}^{t} \theta(\xi) \mathrm{d} \xi\right| \\
& \leq \frac{(1-\eta)}{\mathscr{M}(\eta)}|\theta(t)|+\frac{\eta}{\mathscr{M}(\eta)} \int_{0}^{t} \theta(\xi) \mathrm{d} \xi \\
& \leq \Delta \varepsilon .
\end{aligned}
$$

Theorem 4. Under assumption $\mathscr{A}_{2}$, together with Lemma 2, problem (18) is Hyers-Ulam stable if $L_{\Psi}<1$, which yields that our considered system (2) is Hyers-Ulam stable.
Proof. Let $\mathbf{Y} \in X$ be any solution, and $\overline{\mathbf{Y}} \in X$ is a unique solution; then,

$$
\begin{aligned}
|\mathbf{Y}(t)-(\overline{\mathbf{Y}})(t)|= & \left|\mathbf{Y}(t)-\left(\mathbf{Y}_{0}+\left[\Psi(t, \overline{\mathbf{Y}}(t))-\Psi_{0}\right] \frac{(1-\eta)}{\mathscr{M}(\eta)}+\frac{\eta}{\mathscr{M}(\eta)} \int_{0}^{t} \Psi(\xi, \overline{\mathbf{Y}}(\xi)) \mathrm{d} \xi\right)\right| \\
\leq & \left|\mathbf{Y}(t)-\left(\mathbf{Y}_{0}+\left[\Psi(t, \mathbf{Y}(t))-\Psi_{0}\right] \frac{(1-\eta)}{\mathscr{M}(\eta)}+\frac{\eta}{\mathscr{M}(\eta)} \int_{0}^{t} \Psi(\xi, \mathbf{Y}(\xi)) \mathrm{d} \xi\right)\right| \\
& +\mid\left(\mathbf{Y}_{0}+\left[\Psi(t, \mathbf{Y}(t))-\Psi_{0}\right] \frac{(1-\eta)}{\mathscr{M}(\eta)}+\frac{\eta}{\mathscr{M}(\eta)} \int_{0}^{t} \Psi(\xi, \mathbf{Y}(\xi)) \mathrm{d} \xi\right) \\
& -\left(\mathbf{Y}_{0}+\left[\Psi(t, \overline{\mathbf{Y}}(t))-\Psi_{0}\right] \frac{(1-\eta)}{\mathscr{M}(\eta)}+\frac{\eta}{\mathscr{M}(\eta)} \int_{0}^{t} \Psi(\xi, \overline{\mathbf{Y}}(\xi)) \mathrm{d} \xi\right) \mid \\
\leq & \Delta \varepsilon+L_{\Psi}\|\mathbf{Y}-\overline{\mathbf{Y}}\| .
\end{aligned}
$$

This implies that

$$
\|\mathbf{Y}-\overline{\mathbf{Y}}\| \leq \frac{\Delta}{1-L_{\Psi}} \varepsilon .
$$

Therefore, the solution of (18) is Hyers-Ulam stable. Hence, the solution of the proposed system (2) is Hyers-Ulam stable. 
Remark 2. In the same line, we can also develop the results of generalized Hyers-Ulam, Rassias-Hyers-Ulam stability. The aforementioned stability analysis has been studied for simple mathematical models of biology and physics in [41-43].

\section{Derivation of the General Semianalytical Solution to the Considered Model (2)}

Here, in this section, we are going to compute series solution for the suggested problem. To receive this goal, taking Laplace transform of (16), we have

$$
\left\{\begin{array}{l}
\mathscr{L}[u(t)]=u(0)+\frac{s+\eta(1-s)}{s} \mathscr{L}[\Lambda(1-q)-\beta u(x+y)+\gamma v-\mu u], \\
\mathscr{L}[v(t)]=v(0)+\frac{s+\eta(1-s)}{s} \mathscr{L}[\Lambda(q)+\mu u-\gamma x-\mu x-\omega x], \\
\mathscr{L}[x(t)]=x(0)+\frac{s+\eta(1-s)}{s} \mathscr{L}\left[\beta u(v+y)-\mu v-\alpha v-\mu_{2} v\right], \\
\mathscr{L}[y(t)]=y(0)+\frac{s+\eta(1-s)}{s} \mathscr{L}\left[\alpha v+\delta y-\mu y-\mu_{3} y\right], \\
\mathscr{L}[z(t)]=z(0)+\frac{s+\eta(1-s)}{s} \mathscr{L}\left[\mu_{2} v+\omega x+\mu_{3} x-\mu z\right] .
\end{array}\right.
$$

Now, assuming the solution in the series form,

$$
\begin{aligned}
& u(t)=\sum_{q=0}^{\infty} u_{q}(t), \\
& v(t)=\sum_{q=0}^{\infty} v_{q}(t), \\
& x(t)=\sum_{q=0}^{\infty} x_{q}(t), \\
& y(t)=\sum_{q=0}^{\infty} y_{q}(t), \\
& z(t)=\sum_{q=0}^{\infty} z_{q}(t) .
\end{aligned}
$$

Further decomposing the nonlinear terms $u(t) x(t)$, $u(t) y(t), u(t) v(t)$, etc. in terms of Adomian polynomials,

$$
\begin{aligned}
& u(t) x(t)=\sum_{q=0}^{\infty} A_{q}(u, x), \\
& u(t) y(t)=\sum_{q=0}^{\infty} B_{q}(u, y), \\
& u(t) v(t)=\sum_{q=0}^{\infty} C_{q}(u, v),
\end{aligned}
$$

where the Adomian polynomial $A_{q}(u, x)$ can be defined as

$$
A_{q}(u, x)=\left.\frac{1}{q !} \frac{\mathrm{d}^{q}}{\mathrm{~d} \lambda^{q}}\left[\sum_{j=0}^{p} \lambda^{j} u_{j}(t) \sum_{j=0}^{p} \lambda^{j} x_{j}(t)\right]\right|_{\lambda=0} .
$$

In the same way, the other polynomials $B_{q}, C_{q}$ can be defined.

Hence, in view of (35) and (36), system (34) becomes

$$
\left\{\begin{array}{l}
\mathscr{L}\left[\sum_{q=0}^{\infty} u_{q}(t)\right]=u(0)+\frac{s+\eta(1-s)}{s} \mathscr{L}\left[\Lambda(1-q)-\beta \sum_{q=0}^{\infty} A_{q}(u, x)-\beta \sum_{q=0}^{\infty} B_{q}(u, y)+\gamma \sum_{q=0}^{\infty} v_{q}-\mu \sum_{q=0}^{\infty} u_{q}\right], \\
\mathscr{L}\left[\sum_{q=0}^{\infty} v_{q}(t)\right]=v(0)+\frac{s+\eta(1-s)}{s} \mathscr{L}\left[\Lambda(q)+\mu \sum_{q=0}^{\infty} u_{q}-\gamma \sum_{q=0}^{\infty} x_{q}-\mu \sum_{q=0}^{\infty} x_{q}-\omega \sum_{q=0}^{\infty} x_{q}\right], \\
\mathscr{L}\left[\sum_{q=0}^{\infty} x_{q}(t)\right]=x(0)+\frac{s+\eta(1-s)}{s} \mathscr{L}\left[\beta \sum_{q=0}^{\infty} C_{q}(u, v)+\beta \sum_{q=0}^{\infty} C_{q}(u, y)-\mu \sum_{q=0}^{\infty} v_{q}-\alpha \sum_{q=0}^{\infty} v_{q}-\mu_{2} \sum_{q=0}^{\infty} v_{q}\right], \\
\mathscr{L}\left[\sum_{q=0}^{\infty} y_{q}(t)\right]=y(0)+\frac{s+\eta(1-s)}{s} \mathscr{L}\left[\alpha \sum_{q=0}^{\infty} v_{q}+\delta \sum_{q=0}^{\infty} y_{q}-\mu \sum_{q=0}^{\infty} y_{q}-\mu_{3} \sum_{q=0}^{\infty} y_{q}\right], \\
\mathscr{L}\left[\sum_{q=0}^{\infty} z_{q}(t)\right]=z(0)+\frac{s+\eta(1-s)}{s} \mathscr{L}\left[\mu_{2} \sum_{q=0}^{\infty} v_{q}+\omega \sum_{q=0}^{\infty} x_{q}+\mu_{3} \sum_{q=0}^{\infty} x_{q}-\mu \sum_{q=0}^{\infty} z_{q}\right] .
\end{array}\right.
$$


Now, equating terms on both sides of (38), we have

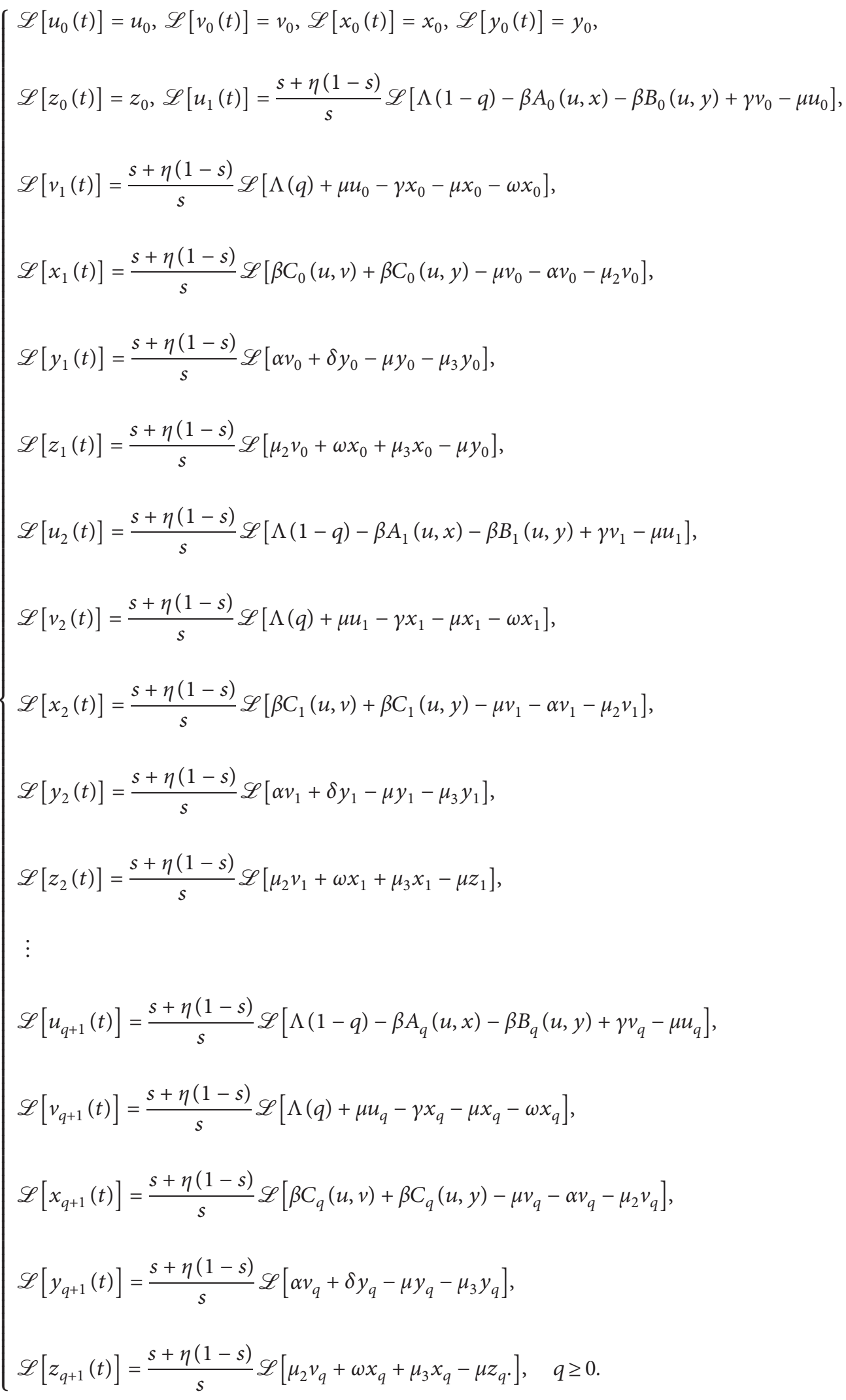


Evaluating the Laplace transform in (39), we get

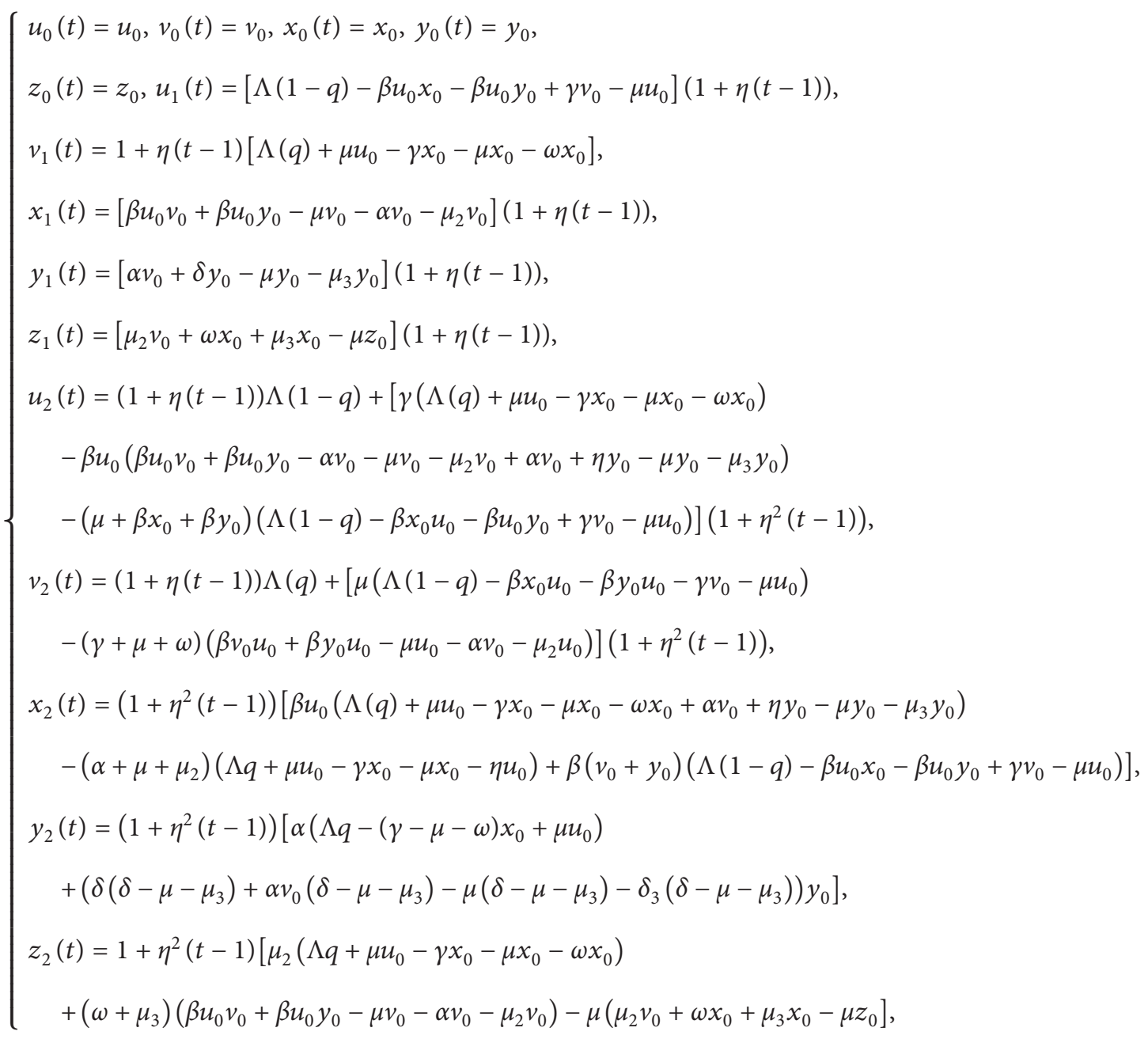

and so on. Therefore, we get the required solution as given by

$$
\left\{\begin{array}{l}
u(t)=u_{0}+u_{1}(t)+u_{2}(t)+u_{3}(t)+\cdots \\
v(t)=v_{0}+v_{1}(t)+v_{2}(t)+v_{3}(t)+\cdots \\
x(t)=x_{0}+x_{1}(t)+x_{2}(t)+x_{3}(t)+\cdots \\
y(t)=y_{0}+y_{1}(t)+y_{2}(t)+y_{3}(t)+\cdots \\
z(t)=z_{0}+z_{1}(t)+z_{2}(t)+z_{3}(t)+\cdots
\end{array}\right.
$$

Note. For the convergence of the proposed method, see the paper [27].

\section{Results and Discussion}

This part of the manuscript is related to provide numerical results and some discussion about the approximate solution of the considered problem. For this purpose, we apply the Laplace Adomian decomposition methods for the solution. We select appropriate values for parameters, as we take [3] $u_{0}=400, v_{0}=200, x_{0}=80, y_{0}=10, z_{0}=0$, $\Lambda=0.005711, \quad \beta=0.0001104, \quad \gamma=0.0007791 ; \alpha=0.7791$; $\mu=0.001103, \mu_{2}=0.005728, \mu_{3}=0.00391, \omega=0.002837$, $q=0.002807$, and $\delta=0.007032$. In view of these values, we draw the graph of approximate solutions (40) for first ten terms by using Matlab in Figures 1-5 against various fractional-order derivatives.

From Figures 1-5, we see that the susceptible class population is decreasing with different rates. It decreases with faster speed when the order is smaller as compared with the larger order. Also, the dynamics of the vaccinated class is increasing with different rates due to the fractional order. Also, the exposed population is increasing up, and hence, on using the vaccine, the density of the population of the infected class is decreasing, while the density of the recovered class increases with the same scenario. From all these figures, we concluded that the fractional derivative with exponential kernel can also be used to provide the global dynamics of the considered model. 


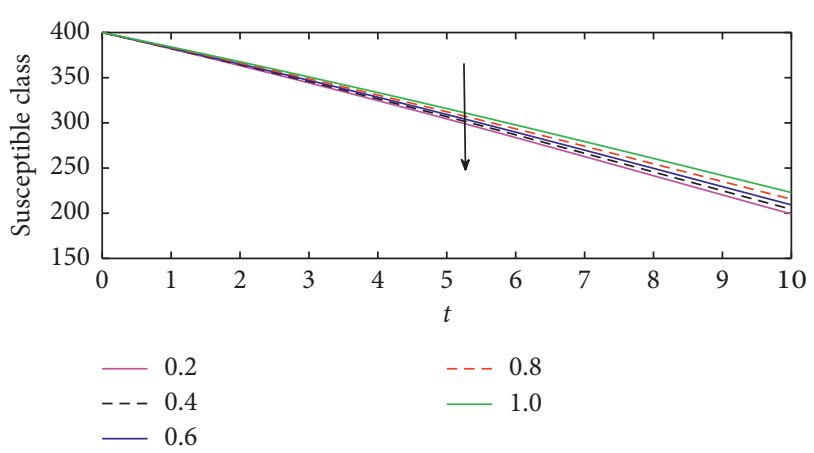

Figure 1: Plot of the susceptible class at different fractional values of $\eta$.

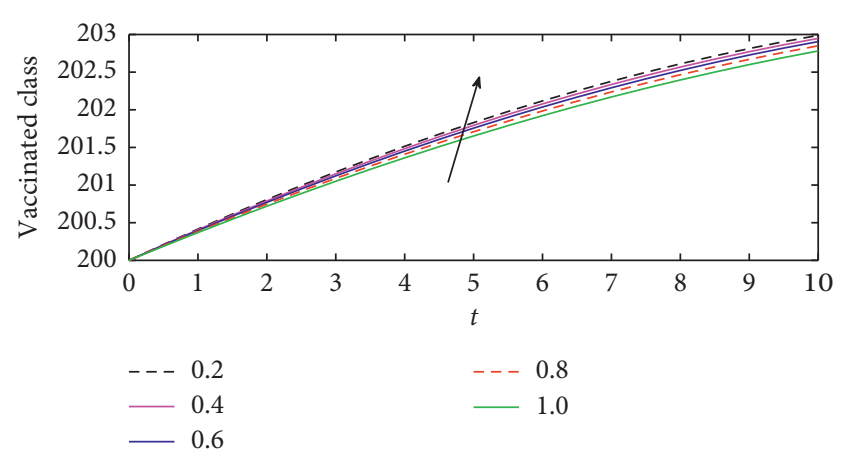

FIgURE 2: Plot of the vaccinated class at different fractional values of $\eta$.

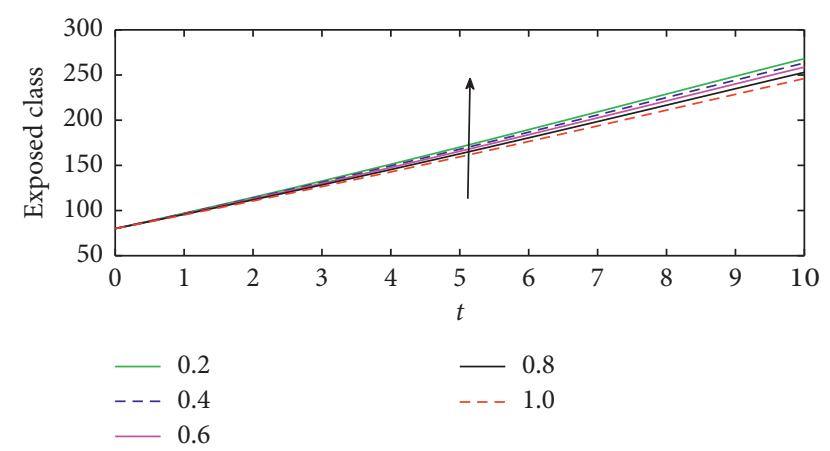

FIgURE 3: Plot of the exposed class at different fractional values of $\eta$.

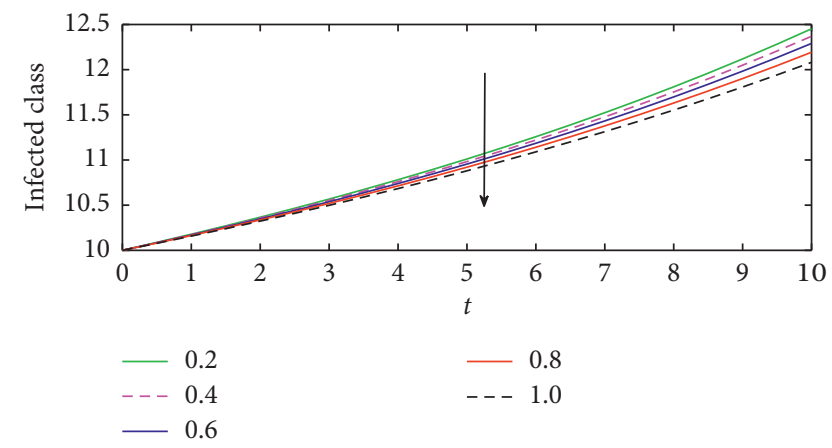

Figure 4: Plot of the infected class at different fractional values of $\eta$.

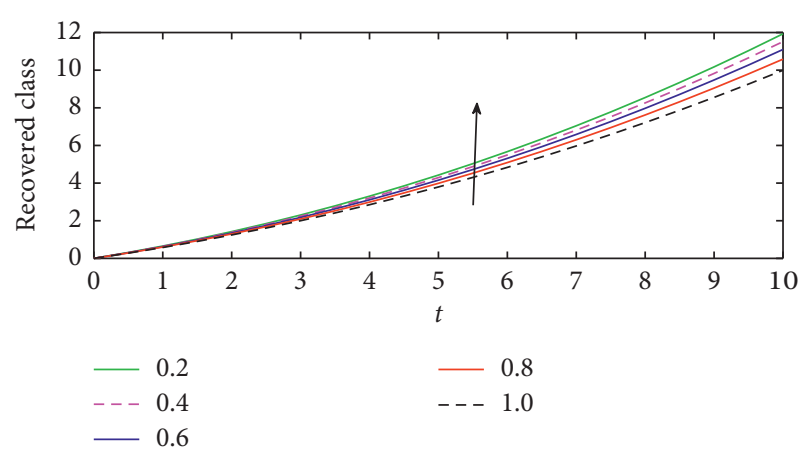

Figure 5: Plot of the recovered class at different fractional values of $\eta$.

\section{Conclusion}

By fixed point approach, we have investigated the existence of the considered model under CFFD. Also, by using the tools of nonlinear functional analysis, we have established sufficient conditions for Hyers-Ulam-type stability of the approximate solutions of the considered model. Also, we have provided the semianalytical solution to the considered model by the Laplace Adomian decomposition method. The concerned method needs no discretization of data nor extra axillary parameter as needed by homotopy methods on which these methods depend. The proposed method has been utilized extensively for usual fractional differential equations, but in the case of new fractional differential operators, this method has not been properly used. Hence, we concluded that CFFD can also be used as powerful tools to investigate biological models of infectious diseases. Also, the concerned models involving CFFD can be handled easily by using the Laplace Adomian decomposition method.

\section{Data Availability}

No data were used to support this study.

\section{Conflicts of Interest}

There exist no conflicts of interest regarding this research work.

\section{Authors' Contributions}

All authors have equal contribution to this work.

\section{Acknowledgments}

The authors are thankful to the HED for supporting this work under grant no. HEREF-46 and HEC of Pakistan under grant no. NRPU-10039. This work was supported financially by the National Natural Science Foundation of China (11971493).

\section{References}

[1] I. Podlubny, Fractional Differential Equations: Mathematics in Science and Engineering, Academic Press, New York, NY, USA, 1999. 
[2] A. A. Kilbas, H. Srivastava, and J. Trujillo, "Theory and application of fractional differential equations," North Holland Mathematics Studies, Vol. 204, Elsevier, Amsterdam, Netherlands, 2006.

[3] M. Caputo and M. Fabrizio, "A new definition of fractional derivative with out singular kernel," Progress in Fractional Differentiation and Applications, vol. 1, pp. 73-85, 2015.

[4] M. Caputo and M. Fabrizio, "Applications of new time and spatial fractional derivatives with exponential kernels," Progress in Fractional Differentiation and Applications, vol. 2, no. 1, pp. 1-11, 2016.

[5] N. Sene, "Stability analysis of electrical RLC circuit described by the Caputo-Liouville generalized fractional derivative," Alexandria Engineering Journal, 2020.

[6] K. M. Saad, "New fractional derivative with non-singular kernel for deriving Legendre spectral collocation method," Alexandria Engineering Journal, 2019.

[7] K. M. Saad, D. Baleanu, and A. Atangana, "New fractional derivatives applied to the Korteweg-de Vries and Korteweg-de Vries-Burger's equations," Computational and Applied Mathematics, vol. 37, no. 4, pp. 5203-5216, 2018.

[8] K. M. Saad, S. Deniz, and D. Baleanu, "On a new modified fractional analysis of Nagumo equation," International Journal of Biomathematics, vol. 12, no. 3, Article ID 1950034, 2019.

[9] H. M. Srivastava and K. M. Saad, "New approximate solution of the time-fractional Nagumo equation involving fractional integrals without singular Kernel," Applied Mathematics \& Information Sciences, vol. 14, no. 1, pp. 1-8, 2020.

[10] H. A. A. El-Saka, “The fractional-order SIS epidemic model with variable population size," Journal of the Egyptian Mathematical Society, vol. 22, no. 1, pp. 50-54, 2014.

[11] T. Hernandez, "A fractional calculus approach to the dynamic optimization of biological reactive systems. Part I: fractional models for biological reactions," Chemical Engineering Science, vol. 117, pp. 217-228, 2014.

[12] Z. Wang, D. Yang, T. Ma, and N. Sun, "Stability analysis for nonlinear fractional-order systems based on comparison principle," Nonlinear Dynamics, vol. 75, no. 1-2, pp. 387-402, 2014.

[13] A. Atangana and J. F. Gómez-Aguilar, "Fractional derivatives with no-index law property: application to chaos and statistics," Chaos, Solitons \& Fractals, vol. 114, pp. 516-535, 2018.

[14] A. Atangana and B. S. T. Alkahtani, "New model of groundwater flowing within a confine aquifer: application of Caputo-Fabrizio derivative," Arabian Journal of Geosciences, vol. 9, no. 1, pp. 1-6, 2016.

[15] H. Khan, J. F. Gömez-Aguilar, A. Alkhazzan, and A. Khan, "A fractional order HIV-TB coinfection model with nonsingular Mittag-Leffler law," Mathematical Methods in the Applied Sciences, vol. 43, pp. 1-21, 2020.

[16] F. Haq, K. Shah, G. ur Rahman, and M. Shahzad, "Numerical solution of fractional order smoking model via Laplace Adomian decomposition method," Alexandria Engineering Journal, vol. 57, no. 2, pp. 1061-1069, 2018.

[17] A. Ali, K. Shah, and R. A. Khan, "Numerical treatment for traveling wave solutions of fractional Whitham-Broer-Kaup equations," Alexandria Engineering Journal, vol. 57, no. 3, pp. 1991-1998, 2018.

[18] O. Kiymaz, "An algorithm for solving initial value problems using Laplace Adomian decomposition method," Applied Mathematical Sciences, vol. 3, no. 30, pp. 1453-1459, 2009.

[19] V. Morales-Delgado, J. F. Gömez-Aguilar, and M. A. TanecoHernandez, "Analytical solution of the time fractional diffusion equation and fractional convection-diffusion equation," Revista Mexicana de Física, vol. 65, no. 1, pp. 82-88, 2018.

[20] J. F. Gömez-Aguilar, H. Yépez-Martínez, R. F. EscobarJiménez, V. H. Olivares-Peregrino, J. M. Reyes, and I. O. Sosa, "Series solution for the time-fractional coupled $\mathrm{mKdV}$ equation using the homotopy analysis method," Mathematical Problems in Engineering, vol. 2016, Article ID 7047126, 8 pages, 2016.

[21] K. M. Saad, M. M. Khader, J. F. Gömez-Aguilar, and D. Baleanu, "Numerical solutions of the fractional Fisher's type equations with Atangana-Baleanu fractional derivative by using spectral collocation methods," Chaos: An Interdisciplinary Journal of Nonlinear Science, vol. 29, no. 2, Article ID 023116, 2019.

[22] J. F. Gömez-Aguilar and A. Atangana, "Fractional HunterSaxton equation involving partial operators with bi-order in Riemann-Liouville and Liouville-Caputo sense," The European Physical Journal Plus, vol. 132, no. 2, p. 100, 2017.

[23] N. Sene and K. Abdelmalek, "Analysis of the fractional diffusion equations described by Atangana-Baleanu-Caputo fractional derivative," Chaos, Solitons \& Fractals, vol. 127, pp. 158-164, 2019.

[24] N. Sene, "Second-grade fluid model with Caputo-Liouville generalized fractional derivative," Chaos, Solitons \& Fractals, vol. 133, Article ID 109631, 2020.

[25] A. A. Alderremy, K. M. Saad, P. Agarwal, S. Aly, and S. Jain, "Certain new models of the multi space-fractional Gardner equation," Physica A: Statistical Mechanics and Its Applications, vol. 545, Article ID 123806, 2020.

[26] K. Shah, M. A. Alqudah, F. Jarad, and T. Abdeljawad, "Semianalytical study of Pine Wilt disease model with convex rate under Caputo-Febrizio fractional order derivative," Chaos, Solitons \& Fractals, vol. 135, Article ID 109754, 2020.

[27] K. Shah, T. Abdeljawad, and F. Jarad, "On a nonlinear fractional order model of dengue fever disease under CaputoFabrizio derivative," Alexandria Engineering Journal, 2020.

[28] R. T. Prey and N. A-Hasley, "The clinical significance of measles; a review," The Journal of Infected Disease, vol. 189, no. 1, pp. 4-16, 2004.

[29] B. P. Prawato, "Stability and simulation of measles transmission model with and without vaccination," For East Journal of Mathematical Sciences, vol. 102, no. 2, pp. 271-281, 2017.

[30] E. O. Oghre and I. I. Ako, "A mathematical model for measles disease," For East Journal of Mathematical Sciences, vol. 54, no. 1, pp. 47-63, 2011.

[31] K. Ejima, R. Omori, K. Aihara, and H. Nishiura, "Real-time investigation of measles epidemics with estimate of vaccine efficacy," International Journal of Biological Sciences, vol. 8, no. 5, pp. 620-629, 2012.

[32] S. Okyere-Siabouh and L. A. Adetunde, "Mathematical model for the study of measles in cape coast metropoli," International Journal of Modern Biology and Medicines, vol. 4, no. 2, pp. 110-113, 2013.

[33] G. Bolarin, "On the dynamical analysis of a new model for measles infection," International Journal of Mathematical Trends and Technology, vol. 7, no. 2, pp. 2231-5373, 2014.

[34] M. Sher, K. Shah, M. Fečkan, and R. A. Khan, "Qualitative analysis of multi-terms fractional order delay differential equations via the topological degree theory," Mathematics, vol. 8 , no. 2, p. $218,2020$.

[35] M. Benchohra and S. Bouriah, "Existence and stability results for nonlinear boundary value problem for implicit differential 
equations of fractional order," Moroccan Journal of Pure and Applied Analysis, vol. 1, no. 1, pp. 22-37, 2015.

[36] H. Khan, A. Khan, W. Chen, and K. Shah, "Stability analysis and a numerical scheme for fractional Klein-Gordon equations," Mathematical Methods in the Applied Sciences, vol. 42, no. 2, pp. 723-732, 2019.

[37] S. Paul, S. P. Mondal, and P. Bhattacharya, "Numerical solution of Lotka Volterra prey predator model by using RungeKutta-Fehlberg method and Laplace Adomian decomposition method," Alexandria Engineering Journal, vol. 55, no. 1, pp. 613-617, 2016.

[38] M. Z. Mohamed and T. M. Elzaki, "Comparison between the Laplace decomposition method and adomian decomposition in time-space fractional nonlinear fractional differential equations," Applied Mathematics, vol. 9, no. 4, pp. 448-458, 2018.

[39] Y. Khan, "An efficient iterated method for mathematical biology model," Neural Computing and Applications, vol. 23, no. 3-4, pp. 677-682, 2013.

[40] Eiman, K. Shah, M. Sarwar, and D. Baleanu, "Study on Krasnoselskii's fixed point theorem for Caputo-Fabrizio fractional differential equations," Advances in Difference Equations, vol. 2020, no. 1, 2020.

[41] E. Ahmed, "On applications of Ulam-Hyers stability in biology and economics," 2010, https://arxiv.org/abs/1004.1354.

[42] A. Atangana and J. F. Gomez-Aguilar, "Decolonisation of fractional calculus rules: breaking commutativity and associativity to capture more natural phenomena," The European Physical Journal Plus, vol. 133, p. 166, 2018.

[43] T. Abdeljawad and D. Baleanu, "On fractional derivatives with exponential kernel and their discrete versions," Reports on Mathematical Physics, vol. 80, no. 1, pp. 11-27, 2017. 